\title{
Fringe Analysis by Fourier Transform Phase-Shifting Method for Four-Beam Moire Interferometry
}

\begin{abstract}
Yuko Yamamoto, Graduate School of Systems Engineering, Wakayama Univ., Wakayama, Japan Yoshiharu Morimoto, Dept. of Opto-Mechatronics, Faculty of System Engineering, Wakayama Univ., Wakayama, Japan Takanori Nomura, Dept. of Opto-Mechatronics, Faculty of System Engineering, Wakayama Univ., Wakayama, Japan Motoharu Fujigaki, Dept. of Opto-Mechatronics, Faculty of System Engineering, Wakayama Univ., Wakayama, Japan Satoru Yoneyama. Dept. of Opto-Mechatronics, Faculty of System Engineering. Wakayama Univ., Wakayama, Japan
\end{abstract}

Four-beam moire interferometry can produce simultaneously two different fringe patterns showing in-plane displacement components $u$ and $v$ along $x$ - and $y$-axes respectively on a specimen. However, the two moire fringe patterns are normally recorded separately. Therefore, it is not easy to measure displacement distributions changing with time. If the two fringe patterns are recorded simultaneously in a single image, it is difficult to separate the two different fringe patterns.

In order to measure the two displacement components, we recently proposed two-directional integrated phase-shifting method. It can separate two different fringe patterns and analyze the two phases of the two fringe patterns.

Furthermore, we proposed another technique for simultaneous analysis using Fourier transform phase-shifting method to improve accuracy of phase calculations. This phase-shifting method is effective to separate the two fringes and to analyze the phases accurately. In the proposed method, we recorded 12 images recording the two fringes simultaneously while the $x$ - and $y$-directional phases are shifted as $6 \pi$ and $8 \pi$ respectively, and extracted the components of frequencies 3 and 4 to calculate the phases of the two fringes.

In this paper, we propose a new technique for simultaneous analysis using only 5 images by the Fourier transform phase-shifting method. According to the Whittaker-Shannon sampling theorem, the sampling frequency should be more than the Nyquist frequency i.e. two times of the fringe frequency. Therefore, in the method mentioned above, we used 12 images, which are more than 8 images obtained by the Whittaker-Shannon sampling theorem. If the sampling frequency is less than the Nyquist frequency, a phenomenon called 'aliasing' appears. As the analyzed frequency is different from the original frequency when aliasing occurs, usually the sampling frequency should be taken so as to be larger than the Nyquist frequency. However, in this paper, we utilize aliasing to calculate the phases of the two fringes with less data sampling.

The Fourier transform phase-shifting method is performed using 3-D image processing. A 3-D $(x, y, \alpha)$ image is formed by many sequential 2-D $(x, y)$ images recorded with phase shifting, where $\alpha$ is the shifted phase value. Two fringe patterns for $u$ and $v$ are recorded in a single image simultaneously. The phases of the two fringes are shifted continuously, and the phase-shifting values are $6 \pi / 5$ and $8 \pi / 5$ per a frame period respectively. After recording 5 images, $\alpha$-directional Fourier transform is performed. The $\alpha$-directional frequency spectrum shows the characteristics of the fringes. Though the frequency of the $x$-directional fringe pattern is 3 , the frequency component of the fringe pattern does not appear at the frequency 3 but -2 , because of aliasing. Also the frequency component of the $y$-directional fringe pattern does not appear at the frequency 4 but -1 . By extracting the frequencies -1 and -2 separately, the two directional phases can be analyzed and the two displacement distributions can be obtained simultaneously.

A thermal deformation of an electronic device is demonstrated using the proposed method applied to moire interferometry. Simultaneous measurement of the two-directional displacement components is performed. 